\title{
Educación sexual integral en las aulas de Argentina: Un análisis de la satisfacción percibida y opiniones de ingresantes universitarios/as
}

\section{Comprehensive sex education in the classrooms of Argentina: An analysis of the satisfaction perceived and the opinions of aspiring university students}

DOI: https://doi.org/10.32870/dse.v0i21.658

\author{
Carolina Cravero* \\ Hugo H. Rabbia** \\ Agostina Giacobino*** \\ Iriana Sartor****
}

\begin{abstract}
Resumen
El presente artículo consiste en un análisis exploratorio de las percepciones, satisfacción, conocimientos y actitudes hacia la educación sexual integral, que presentan jóvenes aspirantes a los estudios universitarios en Argentina. Se propone aportar, desde una perspectiva cuantitativa, la mirada de los/as estudiantes sobre la educación sexual que han recibido en sus trayectos formativos, especialmente de nivel medio.

Los estudios sobre sexualidad muestran que la misma constituye un discurso omnipresente en las instituciones educativas, porque las escuelas no fueron pensadas para abordar este tema, ya que la sexualidad integraba lo privado. Esto ha significado una distancia entre la transmisión de información y su apropiación transformativa por parte de los/as jóvenes. En Argentina, la Ley Nacional de Educación Sexual Integral fue sancionada en 2006. Desde entonces, se indagó sobre su implementación con énfasis en la capacitación docente, pero a partir de la realización de las Pruebas Aprender en 2017, donde se consultó a estudiantes, se comienza a colocar el acento en el reclamo estudiantil por la educación sexual integral. Este trabajo propone adentrarse en la percepción de los/as jóvenes, buscando conocer cuál es el nivel de satisfacción percibida sobre la educación sexual integral que recibieron durante sus trayectos escolares en el nivel medio.
\end{abstract}

Palabras Clave: educación sexual integral - percepción - jóvenes - estudios cuantitativos.

Abstract

TThis article consists of an exploratory analysis of the perceptions, satisfaction, knowledge and attitudes towards the comprehensive sex education of young aspiring university students in Argentina. Through a quantitative approach, it

\footnotetext{
* Doctora en Sociología. CIT Rafaela (UNRaf-CONICET), Santa Fe, Argentina. carol cravero@hotmail.com

** Doctor en Estudios Sociales de América Latina. CONICET-IIPsi, Universidad Nacional de Córdoba y Universidad Católica de Córdoba. Argentina. hugo.rabbia@gmail.com

*** Doctora en Biología. CONICET-UNRaf, Santa Fe, Argentina. agostinagiacobino@gmail.com

**** Universidad Católica de Córdoba. Argentina. irianasartor@gmail.com
} 
seeks to analyze the student's point of view on the sex education they have received throughout their education, especially in high school.

Studies about sexuality show that it is an omnipresent part of discourse in educational institutions. Schools were originally not intended to address this subject, long regarded as "private", thus creating a gap between the transmission of information and its transformative appropriation by students. In Argentina, the Federal Law on Comprehensive Sex Education was passed in 2006, since then, its application has often been researched with an emphasis in teachers training, but after the "Aprender" tests in 2017, in which students were consulted, the stress began to be shifted to the students' demand for comprehensive sex education. This work aims to explore youth perception of sex education, seeking to know the level of satisfaction perceived on the comprehensive sex education received during their years in high school.

Key Words: sexual education - students - perception - quantitative studies.

\section{Introducción}

En el año 2006 se sancionó en Argentina la Ley Nacional № 26.150 de Educación Sexual Integral, que reconoce el derecho a todos los educandos/as de recibir educación sexual integral, tanto en establecimientos escolares de gestión pública como privada. Esta ley, que integra las normas con perspectiva de género del sistema jurídico argentino, es resultado de conquistas de derechos a partir de profundos cambios sociales. Sin embargo, sigue habiendo contradicciones e impedimentos a la hora de su implementación.

La irrupción de las demandas sociales manifestadas por el movimiento de mujeres, de diversidad y colectivos feministas, fueron anclando entre las nuevas generaciones que comenzaron a hacerse eco de reivindicaciones sobre sus cuerpos y la condición sexuada de los mismos, así como del derecho a conocer para poder decidir. En este sentido, la ley creó un programa nacional de Educación Sexual Integral (en adelante ESI) que debe ser impartido en todos los establecimientos educativos, tanto públicos como privados, "desde el nivel inicial hasta el nivel superior de formación docente y educación técnica no universitaria" (Ley № 26.150, 2006, Art. 4).

Si bien la ley obliga a los establecimientos educativos implementar el programa nacional, su artículo 5 establece que "cada comunidad educativa incluirá en el proceso de elaboración de su proyecto institucional, la adaptación de las propuestas a su realidad sociocultural, en el marco del respeto a su ideario institucional y a las convicciones de sus miembros" (Ley № 26.150, 2006). En mayo de 2008, el Consejo Federal de Educación, junto con expertas educativas y referentes de la Iglesia católica, los credos evangélicos y judíos, aprobó los lineamientos curriculares para la educación sexual integral. La implementación de los contenidos establecidos por la ESI, sujetos al ideario institucional, ha generado diversos márgenes de discrecionalidad debido a las interpretaciones que pueden hacerse de este artículo, donde, por ejemplo, varias 
escuelas confesionales redefinieron la orientación de los contenidos con el enfoque de lo que han denominado "educación para el amor", reproduciendo una enseñanza basada en una lógica heteronormativa y patriarcal de la sexualidad, orientada solo a fines reproductivos.

Esquivel (2013) entiende que la educación sexual es parte de aquello que se considera como un "núcleo sensible", que cristaliza los disensos, conflictos y tensiones que se generan dentro de la sociedad y del Estado. El tratamiento legislativo del proyecto de ley de interrupción voluntaria del embarazo, de 2018, puso en agenda como nunca antes el debate acerca de qué educación sexual se debería recibir en las escuelas, generando ciertas tensiones y conflictos entre varios sectores de la sociedad..$^{1}$ En este sentido, en 2018 se presentó un proyecto de modificación de la ley № 26.150, que aún está esperando dictamen parlamentario para su tratamiento. Este proyecto insta a ampliar la perspectiva de derechos y género, incorporando todas aquellas leyes sancionadas posteriores al ESI (por ejemplo, matrimonio igualitario y ley de identidad de género), especialmente, la cuestión referida a la identidad sexual y al género autopercibido es lo que despertó oposición de los sectores conservadores, quienes defienden la tesis de que "los sexos biológicos son dos" (Rafaela Noticias, 21 de junio de 2019).

\section{Fundamentos de la ESI y la noción de integralidad}

La Ley de Educación Sexual Integral surge a partir de una histórica demanda de algunos sectores de la sociedad que cuestionan y disputan el discurso hegemónico de sexualidad, y buscan visibilizar otras prácticas y sexualidades que la heteronorma excluye y deslegitima. Su interés primordial consiste en que "la cultura sexual marginal entre a formar parte de la cultura escolar" (losa, 2008: 2), adoptando un enfoque acorde a las transformaciones de la sociedad. Por otro lado, con la aprobación de la ley de matrimonio igualitario en 2010, comenzó a reconfigurarse el sistema de parentesco, y las escuelas se vieron ante la necesidad de hacer más esfuerzos por incorporar a las familias homoparentales y una perspectiva de no discriminación por orientación sexual. Simultáneamente, la ESI representa una respuesta ante los problemas de salud pública y desigualdades sociales derivados del embarazo adolescente, las infecciones de transmisión sexual, ${ }^{2}$ la iniciación cada vez más temprana en la sexualidad, el aborto (Wainerman y Chimi, 2014), la violencia de género, las identidades de género y las desigualdades de género (Barberis, Taborda y Zamanillo, 2011).

1 Es importante mencionar que durante el debate legislativo, fue común escuchar que quienes se oponían a la ley de interrupción voluntaria del embarazo argumentaron la educación sexual como "solución" al problema de los embarazos no deseados. Sin embargo, una vez finalizado el debate, y que el proyecto no fuera aprobado por la Cámara de Senadores, los sectores conservadores comenzaron a cuestionar la ley de educación sexual integral tildándola de promover la "ideología de género", dando lugar incluso a la campaña transnacional \#ConMisHijosNoTeMetas, que se vio en otros países de la región como Perú, Costa Rica, Colombia y Brasil.

2 De acuerdo con Graciela Morgade (2011), en los años ochenta hubo un avance desde la biologización a lo que la autora denomina "medicalización" a partir de la epidemia de VIH-sida. Esto obligó a las escuelas a abrirse a estos temas y comenzar a poner en discusión cuestiones vinculadas a la sexualidad. 
El enfoque de una educación sexual integral apunta a que la sexualidad sea entendida más allá de la cuestión de la genitalidad o el coito, y se abarquen aspectos biológicos, psicológicos, sociales, afectivos y éticos, una dimensión constitutiva central de la subjetividad y el bienestar humano. El objetivo de esta ley apunta a que se garantice una educación democrática más inclusiva, donde se respete la libertad de las personas a disfrutar de su sexualidad, de acuerdo a sus convicciones y preferencias. En este sentido, la ley establece los siguientes ejes: respetar la diversidad, reconocer la perspectiva de género, valorar la afectividad, ejercer nuestros derechos y cuidar del cuerpo.

La ESI constituye una política pública orientada al cumplimiento e implementación de la perspectiva de derechos humanos incorporada a la Constitución Nacional de la República Argentina desde su reforma en el año 1994, cuando se le otorgó jerarquía constitucional a tratados internacionales como la Convención para la Eliminación de Todas las Formas de Discriminación Contra la Mujer (CEDAW); la Convención sobre los Derechos de los Niños, Niñas y Adolescentes; la Convención Americana de Derechos Humanos, entre otros. Por otra parte, se corresponde con una serie de avances en materia de derechos sexuales y reproductivos que se fueron incorporando al sistema jurídico argentino a partir de 2003, como, por ejemplo, la ley de "parto humanizado" de 2004, la ley nacional de salud sexual y reproductiva de 2006, la ley de matrimonio igualitario de 2010, la ley de identidad de género de 2012, la ley de fertilidad asistida de 2013 y el proyecto de ley de interrupción voluntaria del embarazo que aún no ha sido aprobado.

Varias facciones del ala conservadora y grupos religiosos, como sectores de la Iglesia católica y de algunas iglesias evangélicas, han cuestionado fuertemente la enseñanza de la educación sexual integral y, a su vez, han adoptado una posición cada vez más reactiva frente a las modificaciones propuestas el año pasado a la ley de 2006. Estos grupos sostienen que han de ser los padres quienes ocupen el papel central en cuanto a la formación de sus hijos, y la escuela tan solo debe ocupar un lugar subsidiario; por lo tanto, serían los progenitores quienes poseen derecho a educarlos según sus convicciones y valores (Esquivel, 2013; 2011). A su vez, defienden una educación sexual donde la práctica sexual se limita a la pareja heterosexual estable y monógama, y con fines reproductivos. No existe un libre ejercicio de la sexualidad, como tampoco se reconocen de forma positiva las diversidades sexuales. Su enfoque concibe la sexualidad como "una práctica social que acarrea necesariamente amenazas ante las que han de desplegarse acciones de prevención y control" (Wainerman y Chami, 2014: 133) y la abstinencia es considerada como el método anticonceptivo más eficaz.

La implementación de la ESI pretende ser gradual y progresiva (Wainerman y Chami, 2014). Sin embargo, a 13 años de su sanción, el panorama no parece alentador: en algunos casos se ha recurrido a la ambigüedad del artículo 5 para evadir su propósito original, y en otros, ni siquiera lo acataron. La ley no ocupa un lugar primordial dentro de las agendas de algunas provincias $y$, por ende, no consigue aplicarse en su totalidad a lo largo del territorio nacional. Algunos es- 
tados provinciales no se han adherido al programa de la ESI, ni han desarrollado protocolos de contenidos transversales básicos ni formaciones específicas para sus docentes.

\section{Implementación de la ESI en Córdoba y Rafaela}

Es importante destacar que las ciudades de Córdoba (capital de la provincia de Córdoba) y Rafaela (provincia de Santa Fe), donde se realizó este estudio, se sitúan en una de las regiones más prósperas de la República Argentina, conocida como Región Centro, donde la actividad económica se vincula primordialmente a la producción agroindustrial, se concentra gran parte de la riqueza y cuyo PBI per cápita es $15 \%$ superior a la media nacional. ${ }^{3}$

La unidad de análisis comprende tres universidades, dos de ellas públicas, con la particularidad de que la Universidad Nacional de Córdoba (UCN) es la más antigua del país, y la Universidad Nacional de Rafaela (UNRaf), creada en 2014, la más nueva; y la Universidad Católica de Córdoba (UCC) es una institución privada confesional de gestión jesuita. Se decidió trabajar con aspirantes a distintas dependencias académicas porque, además de ser un universo poco explorado en los antecedentes, la mayoría se encuentran recientemente egresados/as de la escuela media y cuentan con más de 18 años de edad.

En cuanto a la situación de Córdoba, el gobierno provincial se adhirió a la ley nacional, y en 2009 el Ministerio de Educación de la Provincia creó el Programa Provincial Educación Sexual Integral, para aplicarlo en todos los niveles educativos y con contenidos transversales. Ante demandas de un grupo de padres contra lo que consideran un adoctrinamiento en "ideología de género", la resolución ministerial № 433 de abril de 2019, exigió el cumplimiento de la normativa vigente y la implementación de la ESI en todo el ámbito provincial (Boletín Oficial, Provincia de Córdoba, 28 de mayo de 2019).

La implementación de la ESI, sin embargo, no es completamente efectiva. En un relevamiento realizado por el gobierno provincial se advirtió que en el periodo 2013/2015, solo $85 \%$ de las escuelas llevaba a cabo acciones para implementar la ESI, con un incremento en las actividades continuas y planificadas. No obstante, revela también que de la totalidad de los establecimientos encuestados, $42 \%$ presenta la prevalencia de un enfoque moralista, es decir, una educación sexual basada en aspectos morales y religiosos, mientras que en otro $40 \%$ prima una perspectiva integral (Ministerio de Educación, Provincia de Córdoba, 2017).

Estos resultados son similares a los evidenciados en estudios a nivel nacional. A partir de datos recopilados en las Pruebas Aprender 2018, 83\% de las y los estudiantes de quinto y sexto año de nivel secundario consideraron necesario tener más contenidos de educación sexual ( $L a$ Voz del Interior, 14 de noviembre de 2018).

En la Ciudad Autónoma de Buenos Aires, además, los datos recabados en 2017 se complementaron con un relevamiento desde la perspectiva de las/os docentes. En este caso, llaman

3 Fuente: ICEDeL Rafaela. 
la atención las diferencias de percepción en torno a las temáticas que dicen haberse dictado y las que el estudiantado considera que ha recibido en las aulas. Por ejemplo, la formación de prejuicios y estereotipos, la moda y patrones de belleza o la capacidad de decidir con autonomía, son temas que algunos docentes consideran se han dictado más de lo que mencionan las y los estudiantes; mientras que temas como el cuidado del cuerpo y salud reproductiva, la prevención de Infecciones de Transmisión Sexual (ITS), las funciones del aparato reproductor, o los métodos anticonceptivos, son señalados como los más dictados por estudiantes (Ministerio de Educación e Innovación del CABA, 2018).

A su vez, según datos de una encuesta realizada por la Fundación Huésped y el Colectivo por los Derechos Sexuales y Reproductivos, para $86 \%$ de las y los estudiantes la educación sexual se focaliza casi de forma excluyente en cuestiones biologicistas, seguidas de la prevención de embarazos e infecciones de transmisión sexual (72\%) (Fundación Huésped y Colectivo por los Derecho Sexuales y Reproductivos, 2018).

Otro de los obstáculos para la efectiva implementación de la ESI en la provincia de Córdoba, es la poca capacitación de profesores y docentes (Espinosa Miño, 2008; losa, 2013), consecuencia de una falta de articulación entre los mismos y las esferas jerárquicas superiores, lo que deriva en su dificultosa adaptación, ya que son ellas y ellos quienes deben aplicar las indicaciones del lineamiento curricular en las aulas. Adicionalmente, la capacitación implica un gran costo, debido a que la mayoría de las veces son los docentes quienes deben financiarlo de manera particular.

El caso de la ciudad de Rafaela es un poco más complejo, por haber sido declarada Ciudad Pro-vida y Pro-familia en el año 2011, por el órgano legislativo local. Este tipo de adscripciones se debe a iniciativas presentadas por sectores pentecostales y católicos conservadores que se han constituido en grupos de presión política a través de una ONGenización ${ }^{4}$ de lo religioso. Este año, en el contexto electoral por el cual se disputaba la intendencia (alcaldía) de la ciudad, las agrupaciones "Rafaelinos por la vida" $y$ "Rafaelinos por la familia" consiguieron que todos los candidatos al cargo firmaran una carta de adhesión, en la cual se comprometían, entre otras cosas, a reconocer el derecho de los padres a "participar en la elaboración del proyecto institucional del establecimiento educativo a los cuales concurren sus hijos; en base al criterio de que el Estado debe 'asistir y completar' la educación sexual impartida por los padres" (Rafaela Noticias, 21 de junio de 2019), lo cual es contrario a la ley nacional. Por otra parte, el documento pretende que se les reconozca a los padres la potestad de "escoger libremente el tipo de educación que sus hijos habrán de recibir, basada en conocimientos científicos validados, sobre los cuales existe acuerdo suficiente en la comunidad científica, que:" 4.1 responda a su cultura y raíces más

4 Este neologismo es utilizado para dar cuenta de la creación de diversas ONG y organizaciones civiles vinculadas a las iglesias católica y evangélicas que buscan presentarse públicamente como "laicas", y desde allí poder intervenir en los asuntos públicos de una manera más efectiva. En la ciudad de Rafaela son ejemplo de ello las agrupaciones "Rafaelinos por la vida" y "Rafaelinos por la familia".

Diálo Dos 
profundas (dos sexos biológicos)". Esta última cuestión, referida a la identidad sexo-genérica como "autopercibida", es lo que despierta mayores cuestionamientos de los sectores conservadores. Frente a esta situación, tanto docentes de la localidad de Rafaela, aglutinados en el "Frente ESI Rafaela", así como representantes estudiantiles de escuelas secundarias, requirieron audiencia con el intendente de la ciudad y solicitaron el cumplimiento de la normativa vigente.

La provincia de Santa Fe, a diferencia de Córdoba, intentó que la Ley 26.150 se implementara mediante una legislación provincial propia, incorporada a la ley de educación provincial, buscando transformar la ESI en política de estado. Sin embargo, la oposición de fuerzas políticas conservadoras cada vez más visibles en la provincia, junto con el consejo de pastores de la ciudad de Rafaela, hicieron que la iniciativa fracasara en tres oportunidades. De esta manera, al igual que en Córdoba, Santa Fe se adhirió a la ley nacional y el Ministerio de Educación de la Provincia creó el Programa Provincial de Educación Sexual Integral, para ser aplicada en todos los niveles educativos con contenidos transversales.

Es importante destacar que la discusión en torno a la ley provincial polarizó las posiciones acerca de la ESI en la provincia de Santa Fe, haciendo que las iglesias recrudecieran su postura, y que algunas candidaturas políticas en las últimas elecciones incorporaran en su agenda el tema de la educación sexual.

\section{Problema de investigación y objetivos}

Nuestro problema de investigación surge en dos planos, uno empírico y otro normativo. En relación con el primero, constatamos que son escasos los trabajos que indaguen acerca de la educación sexual desde el punto de vista de los y las estudiantes argentinos, especialmente desde una perspectiva cuantitativa. Entre los estudios cualitativos podemos mencionar los trabajos de Graciela Morgade (2011) y Mario Margulis (2003), en los que la escuela aparece como un ámbito donde la sexualidad está presente en todas partes, precisamente por haberse configurado como un asunto que no le correspondía a la institución educativa por pertenecer al "ámbito de lo privado". Durante mucho tiempo, el tema fue marginal en los contenidos curriculares, pero omnipresente entre los y las jóvenes, lo que hizo suponer que estos "sabían más" que las/os encargados de enseñarles. En la década de 1980, con la irrupción del VIH-sida como epidemia y problema de salud pública, se puso de manifiesto que no era necesariamente así, y las escuelas comienzan a aproximarse cada vez más al abordaje de la cuestión, pero siempre desde una perspectiva predominantemente biologicista en la que primaba "la reproducción de la vida humana" (Morgade, 2011: 11), incluso desde visiones moralistas.

También deben señalarse los abordajes con estudiantes y docentes de una escuela confesional de Córdoba, sobre las concepciones de la diversidad sexual y sus modos de apropiación en las aulas (Tomasini, Bertarelli y Estevez, 2017). Las autoras advierten que en dichos contextos, con matices, modulaciones y particularidades, se presenta más una presunción de heterosexua- 
lidad obligatoria y aquello que se aleja de la heteronorma se configura como "anomalía", "rareza" o"caso" (Tomasini et al., 2017: 5).

En el plano normativo, hemos delineado cómo a partir de los avances jurídicos en materia sexual y reproductiva del siglo XXI, la ESI se constituyó en un derecho del estudiantado argentino, y sus contenidos deberían haberse incorporado, al menos a lo largo de la última década, a todos los proyectos institucionales del sistema educativo. Frente a ellos nos preguntamos ¿qué tienen para decir las/os ingresantes universitarios de la Facultad de Psicología de la UNC, la Facultad de Ciencia Política y Relaciones Internacionales de la UCC y de la UNRaf (pública, laica), sobre la implementación de la educación sexual integral en sus trayectos de formación de nivel medio? Interesa aquí, por lo tanto, avanzar hacia una exploración de medidas de satisfacción sobre los contenidos recibidos, así como poder describir con mayores elementos cómo se configura esta satisfacción o insatisfacción sobre la formación en ESI.

De esta manera, nos proponemos explorar las percepciones de ingresantes universitarios sobre los contenidos de ESI, así como la valoración que tienen sobre los mismos (satisfactoria, no satisfactoria o que no resultan pertinentes), y las actitudes genéricas sobre la implementación de la ESI en escuelas medias. Nos interesa especialmente analizar las percepciones, conocimientos y actitudes sobre contenidos de ESI en virtud de la trayectoria de formación de ingresantes (tipo de gestión de las escuelas medias a las que asistió), religiosidad y género. Con ello esperamos contribuir con propuestas, y su efectiva implementación, que permitan avanzar.

\section{Metodología}

El presente trabajo se desprende del proyecto de investigación "¿De ESI se habla?: Un análisis exploratorio de conocimientos, percepciones y actitudes hacia la Educación Sexual Integral en ingresantes universitarios de Rafaela, región, y Córdoba", aprobado en la segunda convocatoria de la Secretaría de Investigación de la Universidad Nacional de Rafaela, para desarrollar un estudio de tipo exploratorio transversal, a partir de datos cuantitativos, acerca de las percepciones y satisfacciones sobre los contenidos de educación sexual impartidos en la escuela media.

\section{Participantes}

Se diseñó un cuestionario semiestructurado para aplicarse a una muestra de conveniencia a 261 estudiantes que aspiran a ingresar a la Facultad de Psicología de la UNC (pública, laica), la Facultad de Ciencia Política y Relaciones Internacionales de la UCC (privada, confesional) y al Ciclo de Formación General ${ }^{5}$ de la UNRaf (Pública, laica). En primer lugar, se decidió trabajar con ingresantes universitarios dado que, en su mayoría, son mayores de 18 años que pudieden par-

5 Se trata de un ciclo básico común para todas las carreras de la Universidad que comprende dos talleres: Taller de lectura y escritura académica, y Taller de estadística y cálculo; y dos disciplinas: Universidad, Estado y Conocimiento, y Problemática contemporáneas, abarcando desde aspirantes a la carrera de Diseño Industrial, pasando por Ingeniería en Computación hasta la innovadora Diseño y Programación de Videojuegos. 
ticipar del estudio sin requerir autorización de padres o tutores. En segundo lugar, se consideró pertinente evaluar las percepciones y satisfacción de quienes hayan atravesado todo el ciclo de formación orientada que representa los últimos años del nivel de educación secundaria, para que pudiera realizarse una exploración ex post facto, a la vez más integral, de las percepciones sobre los contenidos de ESI que desarrollaron en su trayecto previo de formación ya finalizado. Esto implica un sesgo sociodemográfico en términos de las personas participantes, que claramente no representan al universo global de egreso de la escuela media en Argentina. En tercer lugar, se trató de un muestreo de conveniencia por accesibilidad, ya que las/os integrantes del equipo de investigación se desempeñan en las instituciones universitarias en donde se desarrolló el estudio. Esto permitió tener información para poder aportar a la discusión sobre instancias de formación y planificación curricular de ambas instituciones, así como poder generar instancias de debate y discusión posteriores a la toma del cuestionario con el estudiantado.

La muestra final estuvo compuesta por $44.8 \%$ de varones, $54.1 \%$ de mujeres, $0.8 \%$ que se identificó con "Otro" en la respuesta sobre género, y el resto que prefirió no contestar dicha pregunta. De las personas encuestadas, 51.7\% asiste a la UNRaf, en la ciudad de Rafaela, provincia de Santa $\mathrm{Fe}$, y el resto son ingresantes de las dos universidades de Córdoba: de la UCC, $17.2 \%$ y de la UNC, 31\%. Las edades de los participantes fluctúan entre los 18 y 43 años, con una media global de 20.79 años, aunque la media de edad por universidad varía: la de la UNC es de 21.12 y de la UNRaf es de 21.45 años. La media de la UCC es de 18.27 años, relativamente menor a las universidades públicas; esto es esperable tratándose de universidades que apelan a diferentes estratos sociales de origen de las/os ingresantes: la UCC es una institución jesuita de gestión privada, más vinculada con sectores medios altos, con trayectorias educativas más convencionales, mientras las otras dos instituciones son públicas, y con una apertura a ingresantes de mayor diversidad social y con mayor cantidad de trayectorias educativas interrumpidas o no convencionales. En el caso de las encuestas aplicadas en Córdoba, al realizarse en cursos de ingreso de sendas facultades (Ciencia Política y Relaciones Internacionales de la UCC, y Psicología de la UNC) existe menor dispersión en las carreras a las que optan, siendo del ámbito de las Ciencias Sociales y Humanas. En UNRaf, en cambio, se incluyen aspirantes a una gran diversidad de carreras que brinda esta universidad de reciente formación y que cursan un ciclo de formación general integrado, incluyendo ingresantes a la Licenciatura en Administración y Gestión de la Información, Licenciatura en Gestión de la Tecnología, y Licenciatura en Relaciones del trabajo, entre otras.

\section{Instrumentos}

Escala de satisfacción percibida de la formación en ESI en escuela media. Se construyó una escala ad hoc que recuperó de manera explícita - aunque con adaptaciones mínimas para su mejor comprensión - 27 de los contenidos mencionados por el programa nacional de ESI, tanto para 
las orientaciones en ciencias sociales como para ciencias naturales, algunos señalados como lineamientos generales y otros específicos de Educación para la Salud, Ética y Ciudadanía, Psicología, Lengua, Filosofía y Educación Artística.

Entre los contenidos relevados, se incluyeron, por ejemplo: la reflexión y valoración de las relaciones interpersonales con pares, con adultos y con los hijos e hijas; el conocimiento de normas y leyes que tienden a garantizar los derechos humanos del ámbito internacional; la valoración de las relaciones de amistad y de pareja; la identificación de prejuicios y sus componentes valorativos, cognitivos y emocionales; el conocimiento de enfermedades de transmisión sexual, y la promoción de actitudes de cuidado de la salud y hábitos de prevención de las infecciones de transmisión sexual; la reflexión en torno a las implicaciones del embarazo en la adolescencia; la comprensión de los cambios en las configuraciones familiares a lo largo de la historia; el conocimiento de diversos aspectos de atención de salud sexual y reproductiva: los métodos anticonceptivos y de regulación de la fecundidad, entre otros.

Para cada contenido mencionado, las personas participantes del estudio debían responder si lo habían visto, no lo habían visto o no lo consideraban un contenido pertinente de ESI; si los habían visto, debían señalar si se habían abordado de forma satisfactoria o insatisfactoria, según su percepción. A su vez, se agregaron ocho afirmaciones de control, elaboradas a partir de ejes de agenda pública vinculadas mayormente con argumentos de grupos que se oponen a la inclusión de contenidos de ESI en las escuelas. Por ejemplo, se incluyeron: reivindicaciones de los derechos de las mujeres, luchas del movimiento feminista (en sus diversas olas y referentes) y movimientos sociales por los derechos sexuales y reproductivos; la reflexión y el análisis crítico de la ideología de género; o la identificación del lenguaje como aparato ideológico, lenguaje inclusivo y no sexista, entre otros.

A su vez, se indagaron las actitudes genéricas hacia la educación sexual integral a partir del nivel de acuerdo en una escala tipo Likert de 5 puntos (de totalmente en desacuerdo a totalmente de acuerdo) en torno a dos afirmaciones: "La educación sexual debe ser responsabilidad exclusiva de los padres, y las escuelas y colegios no deberían intervenir" $y$ "La educación sexual integral es un derecho de las/os alumnos, y deben recibirla, aunque sus padres se opongan". En el mismo sentido, se pidió también evaluar la percepción sobre la capacitación de profesores para brindar ESI.

Asimismo, se relevó el tipo de gestión y orientación del colegio secundario del cual se egresó: institución de gestión pública, semiprivada confesional o semiprivada laica. También, edad, género y diversos indicadores de religiosidad personal, incluyendo la creencia en Dios o entidad suprema, religión de crianza, religión actual, importancia de la religión en la propia vida y el literalismo bíblico (nivel de acuerdo ante la afirmación: “La Biblia es un texto sagrado que debe ser tomado de manera literal"). ${ }^{6}$

6 Por razones de espacio y significación no se reportan todas las relaciones de las variables del estudio en el presente trabajo. 
Los análisis presentados a continuación consideraron las frecuencias de respuesta (descripción simple univariada) y análisis de contingencia.

\section{Resultados}

\section{Una educación sexual ¿integral?}

En primer lugar, es importante caracterizar en general los contenidos abordados. Al respecto, existe una marcada diferencia entre las/os ingresantes de ambas localidades. Quienes aspiran a ingresar a la UNRaf mencionan que mayoritariamente han visto todos los contenidos indagados, con excepción de la reflexión crítica en torno a los mensajes de los medios de comunicación, a la par que evalúan con al menos $50 \%$ de respuestas a seis contenidos como vistos de manera satisfactoria. Entre estos destacan la información, prevención y cuidado de ITS (66.2\%), y el respeto de sí mismo/a, del otro/a y la valoración y reconocimiento de las emociones y afectos que se involucran en las relaciones humanas, y la valoración y el respeto por el pudor y la intimidad propia y la de los otros/as, con $59.5 \%$ cada caso.

En las/os ingresantes en Córdoba, el panorama es completamente diferente. Se indica que poco menos de la mitad de los contenidos relevados (13 de 27) fueron vistos en al menos 50\% durante los dos últimos años del trayecto de formación secundaria. De estos, la gran mayoría percibe que se vieron de manera insatisfactoria. Tan solo dos ítems han sido abordados de manera mayormente satisfactoria: $56.5 \%$ indica haber visto satisfactoriamente contenidos relacionados con la información, prevención y cuidado de ITS, y 52\%, sobre el conocimiento del ciclo de embarazo y parto. En contraste, se reportan 14 ítems de contenidos que se perciben como mayormente no abordados: $71 \%$ señala no haber visto contenidos sobre análisis, identificación y consecuencias de situaciones de incesto, abuso sexual infantil, pornografía y trata de personas; $65.9 \%$ sostiene que no se les ha enseñado acerca de la legislación existente de salud reproductiva y procreación responsable; $64 \%$ no ha tenido abordaje alguno relacionado con las críticas a las prácticas basadas en prejuicios de género, como tampoco acerca del conocimiento de organismos protectores de derechos en su entorno. Además, 53\% de los/as participantes señala que no han recibido contenidos que trabajen la identificación de la sexualidad como elemento constitutivo de la identidad.

En términos generales, se advierte una predominancia de contenidos de las tradiciones biomédicas, aunque los mismos no son completos, así como de algunos contenidos éticos y vinculares que pueden - aunque no de manera excluyente- abordarse desde una tradición moralizante. Aspectos referidos a los derechos, el análisis crítico de las transformaciones sociales y, sobre todo, contenidos referidos a la valoración de la diversidad sexual, resultan los menos abordados según las/os encuestados.

A continuación, se realiza un recorrido sobre las referencias a diversos ejes de contenidos, la satisfacción percibida respecto a la formación recibida, destacando diferencias significativas 
entre los mismos, si fueron abordados en escuelas públicas o privada, laicas o confesionales, y si son mencionados en Rafaela o en la ciudad de Córdoba.

Tabla 1. Contenidos sobre Anatomía, ITS y anticoncepción.

Percepción de implementación y satisfacción

\begin{tabular}{|c|c|c|c|c|c|c|}
\hline No & Ítem & $\begin{array}{l}\text { Vió } \\
\text { satisfacto- } \\
\text { riamente } \\
\%\end{array}$ & $\begin{array}{l}\text { Vió } \\
\text { insatisfacto- } \\
\text { riamente } \\
\%\end{array}$ & $\begin{array}{l}\text { No vió } \\
\%\end{array}$ & $\begin{array}{l}\text { No } \\
\text { perti- } \\
\text { nente } \\
\%\end{array}$ & $\%$ \\
\hline 22 & $\begin{array}{l}\text { El conocimiento de enfermedades de } \\
\text { transmisión sexual, y la promoción de ac- } \\
\text { titudes de cuidado de la salud y hábitos de } \\
\text { prevención de las infecciones de transmi- } \\
\text { sión sexual (incluido el VIH-sida). }\end{array}$ & 61.5 & 29.2 & 9.3 & 0 & 100 \\
\hline 25 & $\begin{array}{l}\text { El conocimiento anatómico y fisiológico en } \\
\text { las diferentes etapas vitales. }\end{array}$ & $43 \cdot 3$ & 32.9 & 23.0 & 0.8 & 100 \\
\hline 26 & $\begin{array}{l}\text { El conocimiento y la reflexión sobre fecun- } \\
\text { dación, desarrollo embriológico, embara- } \\
\text { zo y parto. }\end{array}$ & 52.1 & 28.6 & 18.9 & 0.4 & 100 \\
\hline 29 & $\begin{array}{l}\text { El conocimiento de diversos aspectos de la } \\
\text { atención de la salud sexual y reproductiva: } \\
\text { los métodos anticonceptivos y de regula- } \\
\text { ción de la fecundidad. }\end{array}$ & 48.4 & 35.9 & $15 \cdot 3$ & 0.4 & 100 \\
\hline
\end{tabular}

En primer término, los contenidos referidos a aspectos anatómicos, fisiológicos o relacionados con ITS, en general manifiestan son los más abordados a lo largo de la escuela media, a la vez, los que más se consideran pertinentes en términos comparativos (o recogen menores referencias a su "no pertinencia") (véase tabla 1).

Es posible advertir diferencias entre la escuela laica y la confesional, especialmente en el ítem 29, conocimiento sobre métodos anticonceptivos y de regulación de la fecundidad. Mientras que $50 \%$ de egresados/as de las escuelas públicas y privadas laicas consideran que los han visto satisfactoriamente, esta posición es evidenciada por solo 38.9\% de egresados/as de la escuelas confesionales. También, uno de cada tres egresados/as de escuelas confesionales manifiestan no haber visto contenidos relacionados con el conocimiento anatómico y fisiológico en las diferentes etapas vitales (ítem 25), porcentaje mayor al de egresados/as de las escuelas laicas (públicas y privadas).

No existen diferencias significativas entre ingresantes en Rafaela y en Córdoba, aunque todos los contenidos son mencionados como más vistos y de forma más satisfactoria entre las y los primeros. 
Tabla 2. Contenidos éticos y relacionales. Percepción de implementación y satisfacción

\begin{tabular}{|l|l|l|l|l|l|l|}
\hline № & \multicolumn{1}{|c|}{ Ítem } & $\begin{array}{l}\text { Vió satisfac- } \\
\text { toriamente } \\
\%\end{array}$ & $\begin{array}{c}\text { Vió insa- } \\
\text { tisfacto- } \\
\text { riamente } \\
\%\end{array}$ & $\begin{array}{l}\text { No vió } \\
\%\end{array}$ & $\begin{array}{c}\text { No } \\
\text { perti- } \\
\text { nente } \\
\%\end{array}$ & \multicolumn{1}{c|}{$\%$} \\
\hline 1 & $\begin{array}{l}\text { La valoración y el respeto por el pudor y la intimi- } \\
\text { dad propia y la de los otros/as. }\end{array}$ & 47.5 & 26.8 & 24.9 & 0.8 & 100 \\
\hline 2 & $\begin{array}{l}\text { La reflexión y valoración de las relaciones inter- } \\
\text { personales con pares, con adultos y con los hijos e } \\
\text { hijas. }\end{array}$ & 50.0 & 28.9 & 20.7 & 0.4 & 100 \\
\hline 3 & $\begin{array}{l}\text { El respeto de sí mismo/a, del otro/a y la valoración } \\
\text { y reconocimiento de las emociones y afectos que se } \\
\text { involucran en las relaciones humanas. }\end{array}$ & 51.2 & 26.0 & 22.8 & 0 & 100 \\
\hline 8 & $\begin{array}{l}\text { El análisis crítico de las prácticas basadas en prejui- } \\
\text { cios de género. }\end{array}$ & 17.4 & 27.5 & 51.9 & 3.1 & 100 \\
\hline 15 & $\begin{array}{l}\text { La valoración de las relaciones de amistad y de pa- } \\
\text { reja. La reflexión en torno a las formas que asumen } \\
\text { estas relaciones en los distintos momentos de la } \\
\text { vida de las personas. }\end{array}$ & 31.8 & 28.7 & 36.5 & 3.1 & 100 \\
\hline 20 & $\begin{array}{l}\text { La identificación de prejuicios y sus componentes } \\
\text { valorativos, cognitivos y emocionales. }\end{array}$ & 24.7 & 33.5 & 39.8 & 2.0 & 100 \\
\hline 33 & $\begin{array}{l}\text { La identificación de la sexualidad como elemento } \\
\text { constitutivo de la identidad. }\end{array}$ & 23.4 & 22.3 & 48.4 & 5.9 & 100 \\
\hline
\end{tabular}

Los contenidos éticos y relacionales han sido asociados más habitualmente con el respeto y la afectividad. Se considera pertinente también incluir aquellos contenidos que abordan diversos aspectos de los prejuicios de género o sexual, puesto que constituyen procesos típicos de las relaciones intergrupales (véase tabla 2). Asimismo, si bien el ítem 33 podría vincularse también con contenidos abordados en el eje de derechos, se advierte que, en los lineamientos curriculares de la ESI, la noción adquiere un marcado abordaje integral, que supone una perspectiva ética. De allí su inclusión en este apartado.

En la tabla anterior se puede advertir una marcada diferenciación entre los contenidos asociados con la afectividad, el pudor y las relaciones interpersonales entre padres, hijo/as y amistades (ítems 1, 2, 3 y 15), respecto de lo que manifiestan los/as participantes del estudio en relación con el resto de los contenidos abordados aquí (prejuicios y sexualidad como elemento constitutivo de la identidad). Los primeros han sido mayormente vistos satisfactoriamente, según la valoración de las personas encuestadas, especialmente en escuelas públicas y, en el caso del ítem 3, también en las escuelas privadas confesionales. Los segundos, en cambio, se considera que se abordaron de forma mayormente insatisfactoria (como es el caso del ítem 20) o bien, que no se han abordado mayormente (como el ítem 8 , referido a los prejuicios de género). Resulta muy llamativo que casi $6 \%$ de los/as respondientes consideró que no resulta 
pertinente brindar contenidos relacionados con la sexualidad como elemento constitutivo de la identidad, siendo uno de los dos contenidos de todos los abordados que recogió percepciones de no pertinencia semejantes. Este contenido es en particular mencionado como no visto por egresados/as de las escuelas confesionales (54\% de los casos), mientras que egresados/as de las mismas escuelas manifiestan en $58.3 \%$ que tampoco han visto contenidos relacionados con un análisis crítico de los prejuicios de género, aspecto en el que coincide $70 \%$ de egresados/as de colegios de gestión privada laica.

Los ingresantes de Rafaela, en comparación de los de las universidades de Córdoba, mencionan mayor satisfacción relativa en todos los contenidos de este apartado.

Tabla 3. Derechos / normativa. Percepción de implementación y satisfacción

\begin{tabular}{|c|c|c|c|c|c|c|}
\hline No & Ítem & $\begin{array}{c}\text { Vió } \\
\text { satisfacto- } \\
\text { riamente } \\
\%\end{array}$ & $\begin{array}{l}\text { Vió insa- } \\
\text { tisfacto- } \\
\text { riamente } \\
\%\end{array}$ & $\begin{array}{c}\text { No vió } \\
\%\end{array}$ & $\begin{array}{c}\text { No } \\
\text { perti- } \\
\text { nente } \\
\%\end{array}$ & $\%$ \\
\hline 4 & $\begin{array}{l}\text { El análisis y debate sobre las identidades } \\
\text { sexuales desde la perspectiva de los dere- } \\
\text { chos humanos. }\end{array}$ & 24.2 & 29.2 & 43.5 & 3.1 & 100 \\
\hline 6 & $\begin{array}{l}\text { El conocimiento de normas y leyes que } \\
\text { tienden a garantizar los derechos humanos } \\
\text { del ámbito internacional. }\end{array}$ & 33.7 & 34.5 & 31.4 & 0.4 & 100 \\
\hline 9 & $\begin{array}{l}\text { La valoración del derecho de las personas } \\
\text { a vivir su sexualidad de acuerdo con sus } \\
\text { convicciones y preferencias. }\end{array}$ & 22.4 & 25.1 & 49.4 & 3.1 & 100 \\
\hline 10 & $\begin{array}{l}\text { El conocimiento y el análisis de las impli- } \\
\text { cancias de los alcances de los derechos y } \\
\text { responsabilidades parentales frente a un } \\
\text { hijo o hija. }\end{array}$ & 26.5 & 24.5 & 46.3 & 2.7 & 100 \\
\hline 11 & $\begin{array}{l}\text { El conocimiento y el análisis de las impli- } \\
\text { cancias de los derechos y responsabilida- } \\
\text { des de los hijos e hijas frente a los proge- } \\
\text { nitores. }\end{array}$ & $24 \cdot 3$ & 27.5 & $45 \cdot 5$ & 2.7 & 100 \\
\hline 24 & $\begin{array}{l}\text { El conocimiento y la utilización de los re- } \\
\text { cursos disponibles en el sistema de salud } \\
\text { de acuerdo con la Ley de Salud Repro- } \\
\text { ductiva y Procreación Responsable (Ley } \\
\text { 25.673). }\end{array}$ & 20.3 & 22.2 & 55.9 & 1.6 & 100 \\
\hline
\end{tabular}

Respecto a los contenidos vinculados a derechos o al ámbito normativo, si bien de una u otra forma todos los otros contenidos relevados pueden enmarcarse en una tradición normativa, se incluyen aquí los ítems que refieren explícitamente a derechos, responsabilidades y a recursos que provienen de algún corpus normativo específico. Se trata de contenidos que tienden marcadamente a 
presentarse menos en las escuelas medias que aquellos referidos a los aspectos anatómicos, ITS y anticoncepción, y que la gran mayoría de los que hemos enmarcado como éticos y relacionales.

Según la percepción de las/os participantes del estudio, en los casos en que se han visto, la mitad considera que los vió de manera satisfactoria y la otra mitad, de manera insatisfactoria (véase tabla 3). Entre egresados/as de instituciones educativas privadas confesionales se afirma de forma mayoritaria que no han visto contenidos relacionados con el análisis y debate de las identidades sexuales desde la perspectiva de los derechos humanos (ítem 4), la valoración del derecho de las personas a vivir su sexualidad de acuerdo con sus convicciones y preferencias (ítem 9), ni el conocimiento y utilización de recursos disponibles en el sistema de salud de la Ley № 25.673 (ítem 24). Este punto también es mencionado como mayormente no visto en escuelas públicas. Mientras que egresados/as de escuelas de gestión privada laica señalan que mayormente no han visto contenidos relacionados con el análisis de las identidades sexuales desde una perspectiva de derechos humanos (ítem 9).

Los contenidos de este eje se mencionan como sistemáticamente menos vistos por ingresantes en las universidades de Córdoba, respecto de la UNRaf, aunque en estos últimos también se dividen porcentualmente en la mitad respecto a considerar si los han visto de manera satisfactoria o insatisfactoria. El ítem 9 (valoración del derecho de las personas a vivir su sexualidad de acuerdo con sus convicciones y preferencias) resulta también uno de los contenidos relevados que recogen mayor proporción de quienes no lo consideran pertinente en Rafaela (4.5\%).

Tabla 4. Contenidos sobre abusos y violencia sexual. Percepción de implementación y satisfacción

\begin{tabular}{|c|c|c|c|c|c|c|}
\hline $\mathrm{N}^{\circ}$ & Ítem & $\begin{array}{l}\text { Vió sa- } \\
\text { tisfacto- } \\
\text { riamente } \\
\%\end{array}$ & $\begin{array}{l}\text { Vió insa- } \\
\text { tisfacto- } \\
\text { riamente } \\
\%\end{array}$ & $\begin{array}{c}\text { No vió } \\
\%\end{array}$ & $\begin{array}{c}\text { No per- } \\
\text { tinente } \\
\%\end{array}$ & $\%$ \\
\hline 18 & $\begin{array}{l}\text { El análisis e identificación de situacio- } \\
\text { nes de vulneración de los derechos de } \\
\text { los niños, niñas y adolescentes. }\end{array}$ & 35.4 & 37.0 & 27.6 & 0 & 100 \\
\hline 19 & $\begin{array}{l}\text { El análisis, identificación e implican- } \\
\text { cias de situaciones de incesto y abuso } \\
\text { sexual infantil; pornografía infantil; } \\
\text { trata de niñas, de niños, de adolescen- } \\
\text { tes y de jóvenes. }\end{array}$ & 14.1 & 20.4 & 63.1 & 2.4 & 100 \\
\hline 30 & $\begin{array}{l}\text { Conocimiento de los organismos pro- } \\
\text { tectores de derechos de su entorno } \\
\text { (líneas telefónicas, programas especí- } \\
\text { ficos, centros de atención, etc.). }\end{array}$ & 17.5 & 28.8 & 52.9 & 0.8 & 100 \\
\hline 31 & $\begin{array}{l}\text { La indagación, reflexión y análisis crí- } \\
\text { tico en torno a la violencia sexual; la } \\
\text { coerción hacia la "primera vez"; la pre- } \\
\text { sión de grupo de pares y los medios de } \\
\text { comunicación. }\end{array}$ & 19.8 & 30.0 & 47.9 & 2.3 & 100 \\
\hline
\end{tabular}


Las formas de vulneración de los derechos de niños/as y adolescentes son múltiples, pero la violencia sexual constituye una problemática central. En este apartado se consideran específicamente los contenidos referidos a estos aspectos. Destacan por tratarse de contenidos que cuentan con un mayor número de referencias relativas a no haber sido vistos durante la escuela media, en especial los ítems 19 y 30, este último, asociado a brindar herramientas a los y las adolescentes y jóvenes para recurrir a organismos protectores de sus derechos (véase tabla 4). Resulta llamativo que casi 70\% de egresados/as de escuelas privadas confesionales manifestaran que no habían visto contenidos relacionados con organismos protectores de derechos en su entorno, mientras que $90 \%$ de egresados/ as de instituciones educativas privadas no confesionales advirtieron que no habían visto contenidos relacionados con situaciones de incesto, abuso sexual infantil o trata de niñas, niños o adolescentes.

En este caso, son más los/as ingresantes en Rafaela, respecto de aspirantes a universidades en Córdoba, que señalan que han visto los contenidos indagados (aunque mayormente de forma insatisfactoria). Por ejemplo, $64 \%$ de ingresantes en Córdoba mencionan que no vieron ninguna información sobre organismos protectores de sus derechos, mientras que este porcentaje desciende a $42 \%$ en ingresantes de la UNRaf.

Tabla 5. Contenidos sobre creencias y transformaciones sociales. Percepción de implementación y satisfacción

\begin{tabular}{|c|c|c|c|c|c|c|}
\hline No & Ítem & $\begin{array}{l}\text { Vió } \\
\text { satisfacto- } \\
\text { riamente } \\
\%\end{array}$ & $\begin{array}{l}\text { Vió insa- } \\
\text { tisfacto- } \\
\text { riamente } \\
\quad \%\end{array}$ & $\begin{array}{c}\text { No vió } \\
\%\end{array}$ & $\begin{array}{l}\text { No } \\
\text { perti- } \\
\text { nente } \\
\%\end{array}$ & $\%$ \\
\hline 35 & $\begin{array}{l}\text { La comprensión de los cambios en las con- } \\
\text { figuraciones familiares a lo largo de la his- } \\
\text { toria, los roles tradicionales para mujeres } \\
\text { y varones y sus transformaciones a nivel } \\
\text { mundial y en la Argentina. }\end{array}$ & 25.5 & 35.6 & 34.7 & 4.2 & 100 \\
\hline 32 & $\begin{array}{l}\text { La reflexión crítica en torno a los mensajes } \\
\text { de los medios de comunicación social refe- } \\
\text { ridos a la sexualidad. }\end{array}$ & 15.2 & 29.6 & 52.1 & 3.1 & 100 \\
\hline 16 & $\begin{array}{l}\text { La indagación y análisis crítico sobre la } \\
\text { construcción social e histórica del ideal } \\
\text { de la belleza y del cuerpo para varones y } \\
\text { mujeres. }\end{array}$ & 21.3 & 27.6 & 45.7 & 5.4 & 100 \\
\hline 12 & $\begin{array}{l}\text { El conocimiento, reflexión y análisis crítico } \\
\text { referido a las tecnologías de la reproduc- } \\
\text { ción y de intervención sobre el cuerpo. }\end{array}$ & 23.6 & 28.6 & 43.9 & 3.9 & 100 \\
\hline 28 & $\begin{array}{l}\text { La indagación y análisis crítico sobre los } \\
\text { mitos o creencias del sentido común en } \\
\text { torno al cuerpo y la genitalidad. }\end{array}$ & 24.3 & 26.7 & 46.3 & 2.7 & 100 \\
\hline 27 & $\begin{array}{l}\text { La reflexión en torno a las implicancias del } \\
\text { embarazo en la adolescencia. }\end{array}$ & 35.7 & 33.7 & 30.2 & 0.4 & 100 \\
\hline
\end{tabular}


En general estos últimos contenidos, referidos a creencias y a un abordaje crítico de la sexualidad a partir de las transformaciones sociales, tratan aspectos poco vistos o vistos de manera insatisfactoria según la valoración de las personas que participaron en el estudio (tabla 5). En particular, se reporta más de 50\% de menciones a "no vistos" en los ítems 28 (creencias y mitos sobre cuerpo y genitalidad) y 32 (reflexión crítica de los mensajes sobre sexualidad de los medios de comunicación), ambos en egresados/as de escuelas privadas confesionales y laicas, y en el último ítem, también en escuelas públicas. Se trata del único contenido explorado que recoge menciones mayoritarias de no haber sido visto en los tres tipos de instituciones educativas de donde provienen las personas encuestadas.

$50 \%$ de ingresantes en UNRaf señala que no haber visto aspectos de reflexión crítica en torno a los mensajes de los medios de comunicación social referidos a la sexualidad, porcentaje que alcanza $54 \%$ en ingresantes en Córdoba.

Como puede advertirse, en general, parecen existir menores consensos en torno a la pertinencia e implementación satisfactoria de contenidos donde se pone en discusión aspectos vinculados con las identidades sexuales, o se discuten cambios societales (como nuevos modelos de configuraciones familiares). En este sentido, estos contenidos han sido particularmente "atacados" por quienes se oponen a la implementación de la ESI en todas las instituciones educativas.

\section{¿Qué opinan los pibes y las pibas sobre la ESI?}

Como se ha mencionado anteriormente, el texto legal establece que la educación sexual integral es un derecho de los educandos y, a su vez, otorga un lugar primordial a las instituciones educativas. De esta forma, la enseñanza de educación sexual se vuelve una responsabilidad de dichos establecimientos. Sin embargo, ciertos sectores conservadores han cuestionado este principio, afirmando que son los padres los que tienen el derecho de inculcar educación sexual a sus hijos e hijas, y que las escuelas ocuparían solo un rol subsidiario (Esquivel, 2013).

Teniendo en cuenta esta disputa, se ha indagado acerca de las actitudes genéricas que poseen las/os encuestados sobre la ESI. Se les ha preguntado si consideran que la misma es un derecho de los alumnos o si debe ser una responsabilidad exclusiva de los padres (véanse gráficos 1 y 2). 
Gráfico 1. La educación sexual integral es un derecho de las/os alumnos, y deben recibirla, aunque sus padres se opongan

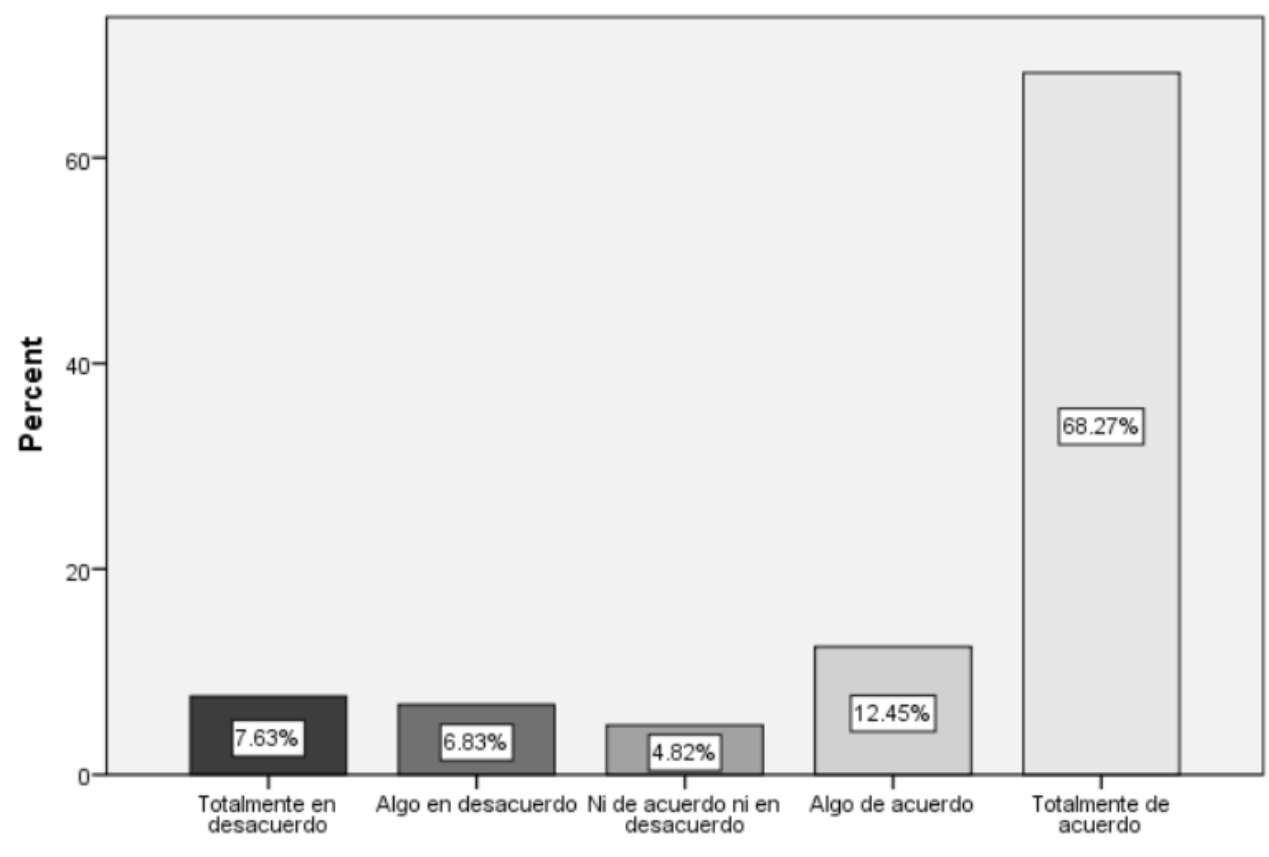

La educación sexual integral es un derecho de los/as alumnos/as, y deben recibirla, aunque sus padres se opongan

Gráfico 2. La educación sexual debe ser responsabilidad exclusiva de los padres, y las escuelas y los colegios no deberían intervenir

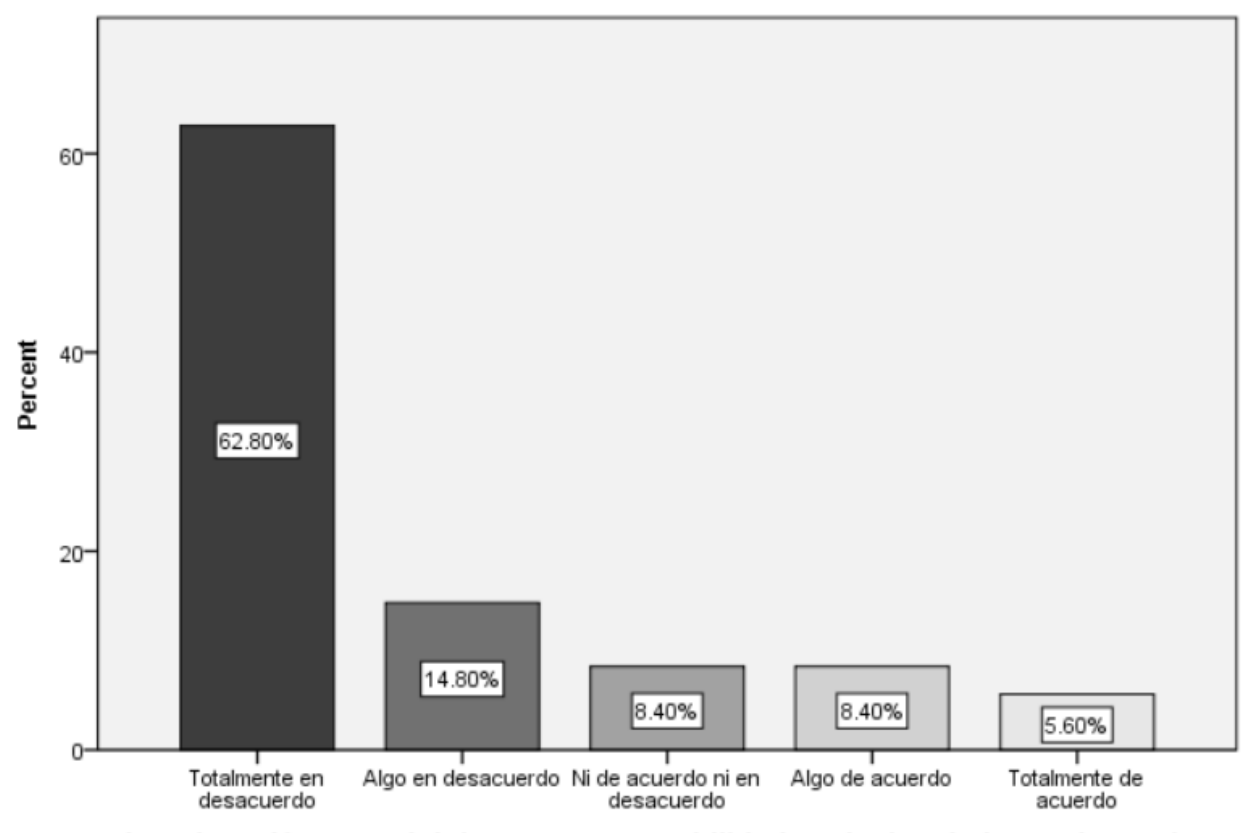

La educación sexual debe ser responsabilidad exclusiva de los padres, y las escuelas y colegios no deberían intervenir 
En general, existen actitudes favorables a considerar que la educación sexual es un derecho de las/os estudiantes, y que deben recibirla incluso cuando sus padres se opongan (algo más de $80 \%$ respondió estar totalmente de acuerdo o algo de acuerdo con la afirmación). En contraste, $77.6 \%$ se mostró totalmente en desacuerdo o algo en desacuerdo con la idea de que la educación sexual es responsabilidad exclusiva de los padres y las escuelas, y colegios no deberían intervenir. Tampoco se advierten diferencias significativas en cuanto al tipo de colegio de procedencia: sean egresados/as de colegios de gestión pública o privada, sean estos confesionales o no confesionales, la gran mayoría de personas encuestadas concuerdan en que la educación sexual es un derecho de las/os jóvenes y que las escuelas deben intervenir, aunque los padres se opongan.

Donde sí se identificaron diferencias significativas es entre varones y mujeres, y según la religiosidad personal. Las mujeres tienden a considerar en $10 \%$ más que los varones, que deben recibir educación sexual integral aunque sus padres se opongan, sin diferencias en el otro enunciado indagado. Católicos y evangélicos coinciden en un porcentaje relativamente mayor en que la educación sexual es una responsabilidad exclusiva de las familias (17\% y $27.5 \%$ respectivamente) respecto de personas sin religión actual (5\%). A su vez, sobre todo evangélicos, manifiestan desacuerdo respecto de que la educación sexual es un derecho de las/os alumnos (34\%), frente a $13 \%$ en católicos y $7.8 \%$ en personas sin religión actual.

Las opiniones generales son algo menos homogéneas al indagar el nivel de acuerdo con el enunciado: “Las/os profesores no están suficientemente capacitados/as para brindar educación sexual integral" (Gráfico 3). Al menos 50\% de los/as encuestadas señaló estar de acuerdo (totalmente o algo) con la afirmación. Aquí se advierten diferencias respecto a si los/as ingresantes provienen de escuelas de gestión pública (donde el nivel de acuerdo alcanza 48\%), respecto a si son de gestión privada, tanto confesional como no confesional (en ambos casos, 56\% de acuerdo). 
Gráfico 3. Las/os profesores no están suficientemente capacitados para brindar educación sexual integral

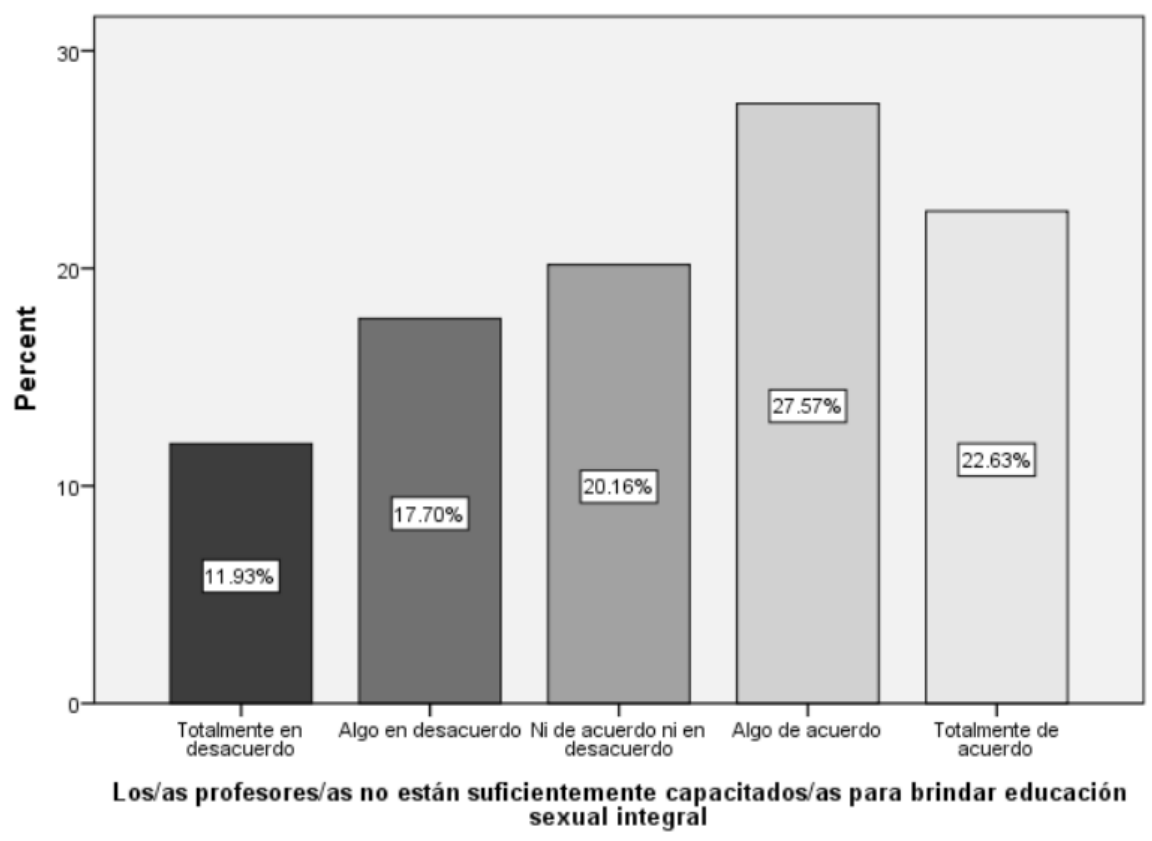

Los/as ingresantes egresados/as de escuelas de gestión pública tienden a ser menos críticos sobre el nivel de capacitación de docentes en torno a contenidos de ESI que los/as egresadas de escuelas de gestión privada (confesional y no confesional). Las mujeres, a su vez, son más críticas que los varones, aunque no es una asociación estadísticamente significativa. Las personas que se identificaron como evangélicas tienen opiniones menos negativas respecto de la capacitación de docentes para brindar contenidos de ESI (42\%, frente a 50\% de católicos y sin religión que señalaron estar algo o totalmente de acuerdo con el enunciado).

\section{Conclusión}

El presente estudio se propuso describir las percepciones y satisfacción sobre contenidos de ESI vistos en las escuelas medias, a partir de un muestreo no probabilístico de ingresantes universitarios en Rafaela y Córdoba, de universidades públicas y privada. Los datos —limitados en su análisis por el carácter no generalizable de la muestra - presentan indicios sobre el bajo nivel de abordaje de los contenidos propuestos por el lineamiento curricular de la implementación del programa nacional de ESI, según la perspectiva de las/os ingresantes universitarios.

Si bien existe una marcada diferencia en las/os ingresantes de ambas localidades, porque quienes se encuentran aspirando a ingresar a la UNRaf mencionan que mayoritariamente han visto todos los contenidos indagados, con algunas excepciones, la mayoría de los ítems que 
expresan mayor satisfacción pertenecen a una perspectiva de corte biologicista o "biomédica" de la educación sexual (Wainerman y Chami, 2014; Morgade, 2011). Sin embargo, no se podría concluir que existe una primacía de dicha perspectiva, debido a que otros contenidos que resultan fundamentales (incluso asociados a las perspectivas biomédicas), no han sido vistos por las/ los ingresantes o no fueron vistos de manera satisfactoria, según su percepción.

Es importante destacar que la diferencia en el nivel de contenidos vistos entre unas y otras universidades debe ser considerado a la luz de que $88.1 \%$ de los aspirantes a ingresar a UNRaf asistieron a escuelas públicas, mientras que en las universidades de Córdoba solo $34.9 \%$ lo hizo, y 53.2\% declaró haber concurrido a escuelas privadas de carácter confesional. En efecto, las principales diferencias porcentuales en las variables indagadas responden a si las/os ingresantes concurrieron a establecimientos educativos de gestión pública o privada (confesional y no confesional). Estas impresiones se refuerzan al considerar que, en términos de actitudes genéricas sobre la ESI, las personas que se identificaron como católicas o evangélicas tienden menos a sostener que la educación sexual es un derecho de las/os alumnos, que las que no tienen o pertenecen a otras religiones, mientras que las evangélicas tienden más a considerar que la educación sexual es una responsabilidad exclusiva de los padres y que los/as docentes se encuentran capacitados para brindar contenidos de ESI que el resto de los/as participantes.

Por otra parte, se encuentra que la gran mayoría (71\% en Córdoba y $60 \%$ en Rafaela) considera que la educación sexual es un derecho del niño, niña o adolescente, mientras que solo una minoría manifiesta total acuerdo en que esta debe ser responsabilidad exclusiva de los padres. Asimismo, se percibe mayormente que los/as docentes no están debidamente capacitados para brindar estos contenidos en las aulas. Si bien no se encontraron relaciones estadísticamente significativas, se evidencian menos contenidos vistos en ingresantes que provienen de escuelas de gestión privada confesional, y en la percepción de mujeres respecto de varones.

En términos de contenidos específicos indagados, se advierte una tendencia a concentrarlos en algunos tópicos de la tradición biomédica (especialmente anatomía e ITS), así como en algunos ejes de contenidos éticos y vinculares (aunque excluyendo los referidos a aspectos relacionados con la identidad sexual como una dimensión constitutiva de la identidad personal, o los referidos a prejuicios de género y sexuales). Los contenidos sobre derechos, abusos y violencias sexuales, y organismos protectores de los derechos de las/os estudiantes son escasamente abordados o abordados de manera insatisfactoria, según los/as encuestadas. A su vez, aspectos relacionados con las transformaciones sociales y las identidades sexuales no heteronormadas siguen siendo tabú, según la percepción de la mayoría de las/os egresados participantes. A su vez, si bien no es recogido por el instrumento, cabe destacar que la insatisfacción percibida por las/os participantes del estudio se debió, mayormente, al carácter moralizante y conservador de algunos de los contenidos brindados en la escuela media, según las impresiones que se pudieron recoger en las discusiones posteriores a la aplicación del cuestionario. 
Cabe señalar que la encuesta fue realizada en un contexto donde el debate por la ESI resurgió en la agenda de los medios de comunicación y la opinión pública. Ciertas organizaciones no gubernamentales y figuras públicas efectuaron reclamos al gobierno nacional por el incumplimiento de la ley de ESI. Al mismo tiempo, el movimiento antiderechos, autodenominado "ProVida", y la campaña \#ConMisHijosNoTeMetas, abogaban en contra de la reforma de dicha ley y la eliminación de la "ideología de género" de los lineamientos curriculares del programa nacional. Por lo tanto, durante el trabajo de campo se advirtió la presencia de un clima tenso y reactivo.

La participación en este estudio fue totalmente voluntaria. Aún así, se han detectado ciertas acciones de protesta o reactivas, por ejemplo: pequeños grupos de personas (en su mayoría varones) que, al escuchar la propuesta del cuestionario, se retiraron del aula, o bien escribieron comentarios dentro del ítem de género que reafirman la binariedad varón/mujer, excluyendo otras formas de autopercibirse, o la decisión de no responder el segundo apartado del cuestionario, que trata sobre la religión. Un caso, por ejemplo, señaló que su religión era "futbolero"; otro caso trazó una flecha que llevaba de las preguntas de religión personal (que no respondió) a las de educación sexual. En parte, este tipo de acciones podrían explicarse por las características del contexto previamente mencionadas y cristalizan una clara posición en este debate, aunque no quedan contempladas en los resultados finales por ser marginales.

En los debates que se sucedieron a la aplicación del cuestionario destacaron, sobre todo, el interés por conocer más sobre la diversidad sexual y sobre las prácticas sexuales desde un abordaje no moralizante. A su vez, se mencionó la necesidad de considerar los procesos sociales y políticos que afectan el orden sexual hegemónico (por ejemplo, los proyectos de legalización del aborto, la expansión del uso de lenguaje inclusivo o la violencia simbólica mediática hacia mujeres y sexualidades diversas) como disparadores a partir de los cuales podrían desarrollarse contenidos pertinentes para el estudiantado. También algunos ingresantes en la UNC (Córdoba) manifestaron la inquietud de no saber de antemano cómo resolver el dilema que les planteaban sus creencias religiosas y las enseñanzas vinculadas con una mirada positiva hacia la diversidad sexual. En el caso de la UNRaf, los grupos de estudiantes que manifestaron interés en el tema, posterior a la aplicación del cuestionario, estuvieron conformados en su mayoría por mujeres. Esta situación implicó la reflexión por parte de las mismas de que la sexualidad continúa siendo entendida socialmente como responsabilidad que recae mayormente en las mujeres, y los varones aún no asumen el compromiso correspondiente. En su conjunto, las reflexiones posteriores generadas por la aplicación del cuestionario permiten reforzar la insatisfacción percibida - evidenciada por el cuestionario - en torno a determinados contenidos que exceden los ejes típicos de la tradición biologicista o de la afectividad.

Como se ha mencionado previamente, el objetivo de este trabajo ha sido realizar un estudio exploratorio sobre las percepciones de los jóvenes acerca de los contenidos impartidos de educación sexual en la escuela secundaria. Sin embargo, otras líneas de investigaciones podrían 
tomar el rumbo de una indagación más profunda acerca de las diferencias existentes entre la educación sexual impartida en las escuelas de gestión pública y de gestión privada confesional, su forma de abordaje y particularidades en la enseñanza; otra opción podría ser un estudio de las percepciones de jóvenes católicos sobre la educación sexual y la posición que adopta la iglesia católica en esta materia, dado que parecen constituir un segmento que no necesariamente responde a las posiciones doctrinarias de la institución. También se podría realizar un estudio de caso sobre la educación sexual que se enseña comparando la situación entre dos o más provincias, en virtud de considerar de qué manera se han adherido (o no) al programa nacional. Asimismo, se podría incluir una triangulación con técnicas cualitativas de construcción de datos, como la entrevista en profundidad a los alumnos, para comprender con mayor hondura qué implica para las/os jóvenes la educación sexual integral (que perciben como deficitaria) y cómo evalúan en términos de satisfacción / insatisfacción la educación sexual recibida.

A su vez, resultaría necesario complementar la información sugerida por nuestro estudio, con un estudio específico de las percepciones de las/os docentes sobre la implementación de los contenidos de ESI en las aulas, ya que, como evidenció el estudio a partir de las Pruebas Aprender en CABA (Ministerio de Educación e Innovación del GCBA, 2018), docentes y estudiantado no necesariamente coinciden respecto a los contenidos que se implementan en las aulas y la satisfacción sobre su formación. Finalmente, consideramos que es urgente indagar con mayor profundidad y con muestreos probabilísticos, las opiniones de las/os jóvenes sobre la ESI en diversos contextos de Argentina. Siendo las/os verdaderos protagonistas del sentido final de la educación sexual integral, resulta particularmente inquietante que sus voces casi no han sido consideradas en decisiones sobre la planificación curricular, las estrategias pedagógicas ni los sentidos involucrados en su propia educación sexual.

\section{Referencias bibliográficas}

Barberis, E.; D. Taborda; A. Zamanillo (2011). Procesos de implementación del Programa Nacional de Educación Sexual Integral en la Ciudad de Córdoba. Análisis de las resignificaciones institucionales del Programa desde un estudio de casos. Síntesis, 4, 1-23.

Espinosa M., Y. (2008) Dislocando saberes y prácticas de la perspectiva de género en educación: de la búsqueda de equidad a la fractura del sujeto educativo. En Colazo, C. (comp.). Deconstruyendo la cultura de género en el sistema educativo cordobés. "Glocalizaciones, dislocamientos y oportunidades". Córdoba: INECIP Centro.

Esquivel, J. C. (2011). El entrevero político-religioso en torno a la educación sexual. Revista Debate Público. Reflexión de Trabajo Social, (1), 45-61.

Esquivel, J. C. (2013). Narrativas religiosas y políticas en la disputa por la educación sexual en Argentina. Cultura y Religión, 7(1), 140-163. 
Fundación Huésped y Colectivo por los Derecho Sexuales y Reproductivos (28 de septiembre, 2017). Resultados de la encuesta sobre Educación Sexual Integral. https://www.huesped. org.ar/noticias/encuesta-educacion-sexual-integral/

Gobierno de la Ciudad Autónoma de Buenos Aires. Ministerio de Educación e Innovación del GCBA. Unidad de Evaluación Integral de la Calidad y Equidad Educativa (2018). Educación Sexual Integral. Encuestas a docentes y estudiantes del nivel secundario. https://www.buenosaires.gob.ar/sites/gcaba/files/ueicee 2018 informe esi 2.pdf

Gobierno de la Provincia de Córdoba (2019). Boletín Oficial de la Provincia de Córdoba. Año CVI, Tomo DCLIII, no 100. Córdoba, (R.A.). https://boletinoficial.cba.gov.ar/wpcontent/4p96humuzp/2019/05/1 Secc 280519.pdf

Gobierno de la Provincia de Córdoba. Ministerio de Educación. Subsecretaría de Promoción de Igualdad y Calidad Educativa (2017). Estado de la implementación de la Educación Sexual Integral en instituciones educativas de la provincia de Córdoba. http://www.igualdadycalidadcba.gov.ar/SIPEC-CBA/PolSocioeducativas/ESI/ImplemESI/Fasciculo\%2003\%20-\%20 SECUNDARIA\%20-\%20\%2017-09-17.pdf

losa, T. (2008). Análisis de aspectos organizacionales del Programa de Educación Sexual Integral. Obstáculos para la ampliación de la ciudadanía sexual en el ámbito educativo. Anuario Digital de Investigación Educativa, (19), 1-11. http://revistas.bibdigital.uccor.edu.ar/index. php/adiv/article/view/3406

losa, T. (2013). Educación sexual e incertidumbre entre el profesorado. Análisis de la implementación del Programa de Educación Sexual Integral en los Institutos Provinciales de Educación Media de la ciudad de Córdoba (Argentina). Voces y silencios. Revista Latinoamericana de Educación, 4(2), 47-61.

La Voz del Interior (14 de noviembre, 2018). El 83\% de las alumnas de escuelas secundarias piden más ESI. https://www.lavoz.com.ar/ciudadanos/83-de-alumnas-de-escuelas-secundariaspiden-mas-esi

Morgade, M. (coord.) (2011) Toda educación es sexual. Hacia una educación sexual justa. Córdoba: La Crujía.

Programa Nacional de Educación Sexual Integral, Ley № 26.150 (2008). Lineamientos curriculares para la Educación Sexual Integral.

Rafaela Noticias (21 de junio, 2019) Declaración para la protección del embarazo vulnerable y la familia. https://rafaelanoticias.com/detalle/68935

Sánchez, C. S. (2017). Actitudes de los docentes de Nivel Medio hacia la implementación de la Ley de Educación Sexual Integral. Anuario de Investigaciones de la Facultad de Psicología, 3(2), 474-487.

Tomasini, M.; P. Bertarelli; M. Estev (2017). Educación y diversidad sexual: Perspectivas de estudiantes y docentes de una escuela confesional de la ciudad de Córdoba, Argentina, sobre 
relaciones homo erótico afectivas. Itinerarus reflectionis, 13(3), 1-23. https://www.revistas. ufg.br/rir/article/view/44800

Wainerman, C.; N. Chami (2014). Sexualidad y escuela. Perspectivas programáticas posibles. Education Policy Analysis Archives/Archivos Analíticos de Políticas Educativas, (22), 127-152. 